R. SAGDEEV,

Space Research Institute

Academy of Sciences

MOSCOW, USSR. read by

A.G. MASSEVITCH.

Mr. President, Ladies and Gentlemen,

I feel very horoured at having been asked by the IAU Executive to present Prof. Sagdeev's invited discourse on venus in his absence. As has already been mentioned Prof. Sagdeev was summoned to the talks between the USSR and the USA in Geneva as an expert in Space Research. He went there with several very important proposals for future cooperation in scientific exploration of outer space and we all hope that the results of his mission will be beneficial for the development of space astronomy and therefore for the whole astronomical community represented by the International Astronomical Union. I am sure that all present in this room realize that it is not an easy task to present someone else's lecture especially at such short notice and in a foreign language.

Besides, it is my opinion that a talk on Venus - the brightest celestial body in the sky except the moon and the Sun - Venus which always has been the symbol of beauty, love and femininity, that such a talk should surely be given by a man.

But realizing the ever increasing trend to shift tasks that have always been considered to be pure men-tasks on the shoulders of women - and this is then called equality ! - I wonder sometimes whether we, women, are not too soft about this. Anyhow, I shall try to do my best and please, be patient with my English.

1. Firstly a very brief historical introduction.

In the pre-telescope era one knew only about Venus that its apparent proper motion was different from that of other stars. Then Galileo found in 1610 that Venus had phases and in 1760 Lomonosov discovered its atmosphere; in the 18th and 19th centuries the presence of dark features or spots on the "surface" of Venus was reported by observers. Venus has been considered a twin sister of Earth since the 1750's when its diameter was measured accurately enough during the passage over the solar disk. The concept of the two "twin planets" was confirmed by the presence of a continuous dense cloud layer enveloping Venus. This cloud layer was, until recently, assumed to consist of water vapour. It is 
only in the twentieth century that most significant results in the astronomical studies of Venus have been obtained with sophisticated technology, i.e. when spectral investigations were started and radio astronomy techniques were first employed. The most essential results of that time are evidently the discovery of carbon dioxide in the atmosphere of Venus, the first estimates of water abundance in the atmosphere layer above the clouds, estimation of traces of carbon oxide, hydrogen chloride and hydrogen fluoride. It is significant to note, however, that even 15 years ago our knowledge of the nature of the clouds was completely obscure. Iron and mercury chlorides, ice and silicate particles were suggested as possible candidates. The density of the atmosphere itself was not known. Neither were the temperature of Venus, its precise mass and even the period and direction of rotation. It should be noted, however, that observations of the drift of dark features over the disk established the presence of atmospheric rotation. Estimates of the rotation period remained contradictory for quite a long time. Even 30 years ago there was a discussion as to whether the period of rotation of Venus might be several days or several weeks. Only radar ranging of Venus permitted us to determine that the rotation was retrograde and that the period was about 243 terrestrial days. Radar ranging also allowed us to determine the radius of the solid body of Venus as being $6050 \mathrm{kms}$. Radio astronomical observations starting in 1957 pointed to very high surface temperatures of the planet. However data obtained at different wavelengths strongly differed. In 1962, investigations of Venus began from outer space. At that time the main astronomical parameters of the planet were determined rather reliably. But the information concerning physical and chemical properties of Venus was still somewhat contradictory, and led often to the construction of mutually exclusive models. The space era crucially changed many of our concepts about Venus. New data on the physical and chemical properties of the atmosphere, abundances, mechanical properties, the images of the planet's surface and other features were obtained by USSR automatic stations Venera-4 to 16, the space crafts Vega 1 and 2 and by the US space vehicles Mariner-5, 10 and Pioneer - Venus. It should be noted that observations from outer space provided most valuable information about the properties of planets of our solar system and created a new branch of science, comparative planetology. Even a brief review and discussion of results obtained during two decades would be a subject for several lectures. Therefore we shall talk here only about the most essential recent results and particularly about the physical and chemical properties of the atmosphere and surface of Venus.

2. Let us start with the atmosphere.

Measurements of temperature and pressure variations with height were carried out with the help of descending probes. Results obtained on space vehicles Venera-7 to 10 established that the surface temperature was about $750^{\circ} \mathrm{K}$ and the pressure about $100 \mathrm{~atm}$. By analogy with our Earth, it was assumed up to 1967 that the major component of the atmosphere of Venus was nitrogen, though as early as in 1930 carbon dioxide absorption lines were recorded in the spectrum of venus. The first spaceborne experiments demonstrated, however, that it is carbon-dioxide 
that is the major component of the atmosphere of Venus, and its abundance was estimated to reach about 96.5 percent. As to nitrogen, it amounts only to 3 percent of the atmosphere, and the remainder are minor components, one of them, sulfur dioxide, being very important for the chemistry of the planet's atmosphere. Its presence was first confirmed by direct measurements from aboard the spacecraft Pioneer-Venus and Venera-12 in 1979. The sulfur dioxide abundances obtained from various experiments agree fairly well, being about 150 parts per million. Below the $40 \mathrm{~km}$ level water vapour is another important minor component of the atmosphere of venus. In the atmosphere above the cloud layer its abundance varies from 0.1 to 0.01 percent according to estimates made by different investigations. New data of interest have also been obtained concerning the presence of gaseous sulfur in the atmosphere of Venus. As to the estimates of abundances for other chemically active minor components, as for instance oxygen, carbon oxysulfide, hydrogen sulfide, they still have to be confirmed and refined.

Data obtained from outer space have already been used to develop a number of interesting photo- and thermochemical models of the atmosphere of Venus, among them several models that take into account interaction between the atmosphere/surface. Data recently delivered by the Vega-1 and Vega-2 spacecrafts proved rather convincingly the presence of $\mathrm{H}_{2} \mathrm{SO}_{4}$ (sulphuric acid) aerosols in the cloud layer of venus. As it happens, new scientific experiments supply not only new information but create at the same time new problems. For instance, preliminary results of the Vega spacecrafts concerning the composition of the cloud-layer aerosols showed that, in addition to sulfuric acid and chlorine, phosphorus is also present in the clouds of Venus. If these data are confirmed it would lead to a most radical revision of our present concepts about the chemistry of the planet's atmosphere-clouds-surface system. It should be noted however that the chemical composition of the aerosols in the planet's cloud layer is still not fully determined.

Another difficult problem is that of the low abundance of water on venus. Available data on the ratio of the hydrogen isotopes $D / H$ (though they do need updating) seem to imply that in the past Venus had much more water. A natural question arises concerning the mechanisms through which Venus could have lost its water. In this respect the following processes could be considered. Water may undergo photodissociation by solar UV radiation; hydrogen will then escape into space and oxygen will interact with the crustal material. Water may also dissociate into hydrogen and oxygen at high temperatures in the interiors of Venus. Oxygen thus formed will react with the magma, and hydrogen will be released into the atmosphere and then escape into space. Water may also react with iron oxide or reduce atmospheric components such as carbon oxide. Still, it is not clear as yet which processes were dominant, and the question of water deficit is still open. Studies of the chemical composition of the atmosphere of Venus using space technology supplied surprising results not only about chemically active minor components. Unexpected data have been obtained for the abundances of isotopes of inert gases in the atmosphere of Venus. Mass-spectrometrical measure- 
ments of argon and neon isotopic abundances on Venus showed that the abundance of non-radiogenic isotopes of these gases is one or two orders higher than that on Earth, while the amount of radiogenic argon is almost the same on Venus and Earth. This extremely important experimental result calls for a thorough analysis of the physical conditions at the time of the formation of the terrestrial planets, since the isotopic ratios of inert gases on Venus drastically differ from those characteristic of solar matter. A steady trend has been revealed : the content of non-radiogenic isotopes of inert gases decreases along the "chain" Venus-Earth-Mars. This is an example how planetary studies from outer space can trigger new cosmogonical concepts about processes that occurred in the protoplanetary nebula billions of years ago. Optical measurements from on board the Pioneer and Venera-15 and 16 orbital stations supplied significant data about the atmosphere of Venus, in particular, about the distribution of thermal currents. Hot spots were revealed near the poles and it appears that the polar regions emit on an average $25 \%$ more energy than regions at lower latitudes. Infrared and ultraviolet spectra indicated also that the abundance of sulphur dioxide is essentially lower above the clouds (approximately, by two orders of magnitude) than that in the subcloud layer. Descending probes succeeded in observing an appreciable electric activity in the atmosphere of Venus which may be associated with the occurence of volcanism on this planet. Interesting results were also obtained while studying the magnetic field in the interplanetary space around venus. Spaceborne experiments convincingly showed that the magnetic moment of venus does not exceed $10^{-4}$ of that of the Earth. The absence of a magnetic field results therefore in a peculiar pattern of the solar wind interaction with the upper atmosphere of Venus, demonstrating rather spectacularly a flow around a non-magnetic obstacle of a planetary scale.

Because the atmosphere of Venus is not protected by a magnet ic field the solar wind penetrates to comparatively dense atmospheric layers. Hence, a narrow ionospheric layer is formed at the day side, at a height of about $100 \mathrm{~km}$ with an electron density of about $10^{5} \mathrm{~cm}^{-3}$. A relation between the absence of a planetary magnetism of Venus and its slow rotation cannot be ruled out of consideration.

3. Let us now discuss the dynamics of the atmosphere.

One of the most striking phenomena of Venus is the fast rotation of its atmosphere with a velocity of the cloud-layer about 50 times that of the planetary surface rotation, the so-called super-rotation of the atmosphere. As has been mentioned earlier, the first indications of an east-west drift of patchy features in the atmosphere of Venus with a velocity of about $100 \mathrm{~m} / \mathrm{s}$ were observed in the ultraviolet in $1961 \mathrm{from}$ earthbound observations. The reality of a fast rotation of the major mass of the Venus atmosphere was proved only by in-situ measurements of the wind velocity made from descending probes from the Venera and Pioneer-Venus spacecraft. An atmospheric super-rotation (insignificant however) was discovered later also on the Earth and other planets as well as on satellites with atmospheres. 
A new stage of the investigations of the dynamics of the atmosphere of Venus began with Vega balloons which carried out measurements during a long time and covered large distances in the cloud layers. Prior to this the atmosphere was sounded with eleven USSR Venera landers and four American Pioneer-Venus probes, each set of data collected during approximately 60 minutes. The Vega balloons operated about 48 hours in the atmosphere and obtained information over 11 thousand $\mathrm{km}$ of their drift. An important feature of this experiment is that not only meteorological instruments on board the gondola were used for measurements but that the balloon itself was comoving with the atmospheric currents; an on-board radiosystem and a ground-based radiotelescope network (VLBI) determining the balloon velocity and its coordinates in the atmosphere. Results after the first analysis of the telemetric data include the following :

- the average velocity of the wind is 6 to $10 \mathrm{~m} / \mathrm{s}$ above that determined in previous experiments;

- there exists different air masses, at latitudes $7^{\circ} \mathrm{N}$ and $7^{\circ} \mathrm{S}, 100$ hours apart in time; the air mass in the northern hemisphere is being warmer by $6.5^{\circ}$;

- long periods (about several hours) of intensive downwellings were recorded when the vertical velocity of the atmosphere reached $3 \mathrm{~m} / \mathrm{s}$;

- the period of the most intensive and longest downwelling coincides with the time when the balloon was drifting over the mountain region Terra Aphroditi with a height difference of about $5 \mathrm{~km}$;

- a possible dependence of the zonal current velocity on local time (sun elevation angle) was noted;

- an appreciable meridional component of the wind velocity in the region of Terra Aphroditi was recorded;

- the cloud layer along the entire drift distance of the first balloon (height 53 to $54 \mathrm{~km}$ ) appears homogeneous;

- there exists a discernible radiant flux from the planetary surface at 1 micron wavelength at the night side;

- comparatively fast transfer from night to day at the morning terminator is noted;

- the illumination changes by a factor $10^{5}$ at a distance of about 800 $\mathrm{km}$.

At present the processing of VLBI measurements of coordinates and the velocity of the two balloons is still under way. The estimated quality of the recorded signals confirms that the data on the meridional and zonal components of the wind velocity will be obtained with the accuracy better than $1 \mathrm{~m} / \mathrm{s}$, and of coordinates about $10 \mathrm{~km}$. These measurements should supply information on the direction of the mean meridional circulation and on the correlation of large-scale pulsations of horizontal and vertical components of the wind velocity.

\section{The surface.}

Radar imaging from the Venera-15 and 16 spacecraft offers radically new possibilities for studying the solid surface of Venus. These experiments are complementary to radar measurements which started with the Pioneer-Venus mission. 115 million square kilometers were imaged with a resolution of 1-2 km. Today only several general results of these 
experiments will be presented as a detailed description will be given by $D_{r}$. Rzhiga at the meeting of Commission 16.

It turned out that the surface of venus is rather flat; about 80 percent of the imaged area is located at the mean planetary level. However, lava flows can be easily seen on plains, which in most cases can be identified with formations of the type of calderas or faults. The determination of the composition of rocks on the surface of venus carried out by Venera-10, 13 and Vega 2 landing probes shows that these rocks are similar to terrestrial rocks and lunar basalts. It seems that the role of areal basalt volcanism in the geological history of the planet is rather significant. The analysis of the images revealed also peculiar ring structures on the surface with a diameter from 200 to 600 $\mathrm{km}$. They are located near Terra Ishtar. Geologists already named them ovoids. It is suggested they are relic hot points of the planet at which uplifting and outcropping of material from the interior occurs. Plato Lakshmi may be such a hot point.

About 150 craters with a diameter from several kilometers to 140 $\mathrm{km}$ were recognized on the surface of the plains and in the mountain regions (within the imaged area). These craters are evidently of impact origin since they are similar to impact craters seen on other planetary bodies. Less prominent formations of impact origin are also revealed. The estimated ages of the above formations allow a conclusion that in the past during a period from 600 million to 3 billion years ago the surface of Venus was intensely reworked. Recently, however, these processes became much slower. In this respect Venus turns out to be more similar to Earth than to the Moon, Mercury or Mars. All these recently obtained results, many of which need further confirmation, convincingly show that a revision of our concepts about the nature of Venus in several aspects is necessary.

Undoubtedly there remain many problems to be solved. Here are several of them :

1. Determination of the chemical composition of the near-surface atmosphere and aerosol in the cloud layer.

2. Study of the UV absorption.

3. The intensity of the volcanic and seismic activity on Venus.

4. The interior structure.

5. Determination of the heat flow from the planetary interior.

6. Nature of the atmospheric super-rotation.

7. The water deficit problem.

Maybe, these problems do not cover the whole range of problems related to studies of Venus, but their solution will allow us to gain additional information necessary for a better understanding of the mysteries of this planet and general problems related to the origin and evolution of the Solar system. 


\section{Conclusion.}

All the data collected till now about Venus are the result of conscientious cooperation of scientists from many countries in the framework of large international projects. For example, during the Venera-11, 12 and Pioneer-Venus missions the activity of both sides have been coordinated very efficiently, the teams kept in touch with each other and the data exchange was organized properly. As a result, the well-known manuscript Venus, prepared together, was published by the Arizona University Press and Venus maps compiled from Pioneer data were used to plan landing sites for USSR probes.

Without a broad international radio-astronomical network it would be impossible to determine the coordinates of Vega-1, 2 balloons with the needed accuracy. The two Vega spacecraft carry instruments developed by scientists of France, Czechoslovakia, Hungary, the German Federal Republic and Austria.

Astronomers all over the world are looking forward to the encounters of the space vehicles with Halley's comet. This too is a large international collaboration. Here you can see how the encounter of the Vega missions with the comet is expected. The important dates are 6 to 9 March 1986. The shortest distance will be $10.000 \mathrm{~km}$; scientific measurement will start two days in advance from a distance of 14 million $\mathrm{km}$, then from a distance $7 \mathrm{million} \mathrm{km}$ and then during 3 hours from 550 thousand till 10 thousand $\mathrm{km}$. A rotating platform equipped with a threechannel and an infrared spectrometers, a mass-spectrometer and a TV camera will be pointed in the direction of the comet. Data from Vega 1 will be transmitted to the ESA mission Giotto in real time starting March 6. All the astronomical observatories will be involved. The International Halley Watch is very efficiently coordinating their efforts. This will be really an exciting time for the astronomical community and I am sure all present here are wishing a successful fulfillment of the tasks of the space missions from the European Space Agency, Japan and the USSR. They are certain to supply us with abundant new data on the physics of comets.

In conclusion I should like to make a remark that is not in the manuscript Prof. Sagdeev gave me, but that he doubtless would have added. I mentioned earlier that Venus has always been regarded as a symbol of beauty, love and womenhood. Do the new discoveries about Venus change this attitude. I am sure they do not. On the contrary, these new results bring the symbol nearer reality.

A very high temperature has been found. But who would like love to be lukewarm ? There are traces of sulphuric acid in the atmosphere - but wouldn't one be very quickly fed up with sugar only - ? Storms in the upper layers : which family is free of minor explosion? And on the average the surface explosions come from the part of the wife and those from the husband's part are much deeper.

And finally, like a perfect woman, Venus dit not reveal all her secrets notwithstanding the powerful attack of so many spacecraft. 